\title{
Controlling turbulence in a surface chemical reaction by time-delay autosynchronization
}

\author{
C. Beta, M. Bertram, A. S. Mikhailov, H. H. Rotermund, and G. Ertl \\ Fritz-Haber-Institut der Max-Plack-Gesellschaft, Faradayweg 4-6, 14195 Berlin, Germany \\ (Received 10 January 2003; revised manuscript received 6 February 2003; published 29 April 2003)
}

\begin{abstract}
A global time-delay feedback scheme is implemented experimentally to control chemical turbulence in the catalytic CO oxidation on a $\mathrm{Pt}(110)$ single crystal surface. The reaction is investigated under ultrahigh vacuum conditions by means of photoemission electron microscopy. We present results showing that turbulence can be efficiently suppressed by applying time-delay autosynchronization. Hysteresis effects are found in the transition regime from turbulence to homogeneous oscillations. At optimal delay time, we find a discontinuity in the oscillation period that can be understood in terms of an analytical investigation of a phase equation with time-delay autosynchronization. The experimental results are reproduced in numerical simulations of a realistic reaction model.
\end{abstract}

DOI: 10.1103/PhysRevE.67.046224

PACS number(s): $05.45 . \mathrm{Gg}, 82.40 . \mathrm{Bj}, 82.40 . \mathrm{Np}$

\section{INTRODUCTION}

Nonlinear dynamics of high-dimensional, spatially extended systems became a subject of intense research interest over the past decade [1]. Among chemical systems, the Belousov-Zhabotinsky (BZ) reaction $[2,3]$ is the most prominent example of a reaction-diffusion system showing a variety of complex spatiotemporal patters such as traveling waves, target patterns, and rotating spiral waves [4]. Besides reactions in aqueous phase, where the detailed mechanism is often complex and sometimes not even fully understood, there are a number of simple heterogeneous catalytic reactions where pattern formation has been intensively studied [5]. The catalytic oxidation of $\mathrm{CO}$ on platinum (110) is the most thoroughly investigated reaction of this type [6]. The mechanism of $\mathrm{CO}$ oxidation on platinum (110) is well established [7], and a simple realistic three-variable model has been developed that accounts for most of the dynamic features of this reaction $[8,9]$. The model was later extended by a fourth variable to include the formation of a subsurface oxygen species $[10,11]$. Also, effects of intrinsic global gas phase coupling on the dynamics of the reaction have been investigated in detail [12-14].

In the field of nonlinear dynamics, control of dynamical behavior, in particular, control of chaos is a key issue of recent research [15]. The question of chaos control has first been addressed for low-dimensional systems in a work by Ott, Grebogi, and Yorke (OGY) [16]. Their work has inspired a large number of theoretical as well as experimental studies of feedback control to nonlinear dynamical systems (see, e.g., Refs. [17-22]) and has been extended to address issues of control in spatially distributed systems [23]. The OGY method stabilizes unstable periodic orbits embedded in the chaotic attractor of the system by applying small timedependent perturbations. It is, however, restricted to relatively slow phenomena since permanent extensive computer analysis of the system state is required. In a much simpler algorithm proposed by Pyragas [24], a continuous control of the system is imposed by a feedback signal, generated from the time series of one of the system variables. The applied feedback $F$ is proportional to the difference between the delayed value of the chosen system variable $u$ and the instan- taneous value of this variable, $F \sim u(t-\tau)-u(t)$. We will refer to this feedback scheme as time-delay autosynchronization (TDAS) [25]. Extensions [26] and considerable improvements $[27,28]$ of TDAS were reported in the recent literature. For spatially extended experimental systems which do not allow local access to their individual elements, TDAS can be applied as a global feedback, where the feedback signal is generated from the integral value of $u$ over all system elements. In previous work, the application of TDAS has been studied, either experimentally or theoretically, for only a few systems such as lasers [29], semiconductors [30], glow discharge devices [31], and populations of electrochemical oscillators [32].

For the catalytic $\mathrm{CO}$ oxidation on platinum, aspects of pattern formation and suppression of chemical turbulence under global delayed feedback have previously been studied for a different feedback scheme [33-35] and can be theoretically interpreted in the more general framework of the complex Ginzburg-Landau equation [36,37]. However, the feedback scheme used in these studies is of an invasive nature, that is, the system is continuously driven by the feedback signal. In contrast to this, TDAS is a noninvasive feedback that drives the system until the desired state is reached and becomes small in the state of control. For this reason, we have carried out a separate investigation on the control of chemical turbulence in the catalytic $\mathrm{CO}$ oxidation using the noninvasive TDAS scheme.

The paper is organized as follows. In Sec. II, the experimental setup and the feedback scheme are introduced. The experimental results are presented in Sec. III. Here, the feedback-induced transition from chemical turbulence to homogeneous oscillations, the effects of hysteresis, and the dependence of the oscillation period on the delay parameter are investigated. In Sec. IV, we develop a theoretical interpretation of the experimental results in terms of a phase dynamics equation. The dynamical behavior of the phase model is analyzed and we compare the experimental results with numerical simulations of the phase equation, on the one hand and a realistic model for the uniform system, consisting of three coupled ordinary differential equations, on the other hand. The paper ends with conclusions and a discussion of the obtained results in Sec. V. 


\section{EXPERIMENTAL SETTING}

The catalytic oxidation of $\mathrm{CO}$ on a platinum (110) single crystal surface proceeds via a Langmuir-Hinshelwood mechanism [7]. Before the catalytic reaction can take place, molecules of both $\mathrm{CO}$ and $\mathrm{O}_{2}$ have to adsorb from the gas phase on the catalytic surface. In contrast to the adsorption of $\mathrm{CO}$, the adsorption of $\mathrm{O}_{2}$ is dissociative. By a reaction of the different adsorbates, carbon dioxide is formed and immediately released into the gas phase. Since the dissociative adsorption of $\mathrm{O}_{2}$ requires two adjacent free sites on the surface, a high $\mathrm{CO}$ coverage asymmetrically inhibits the adsorption of $\mathrm{O}_{2}$. Furthermore, depending on the adsorbate coverage, a phase transition of the $\mathrm{Pt}(110)$ surface between a $1 \times 2$ missing row structure (clean and O-covered surface) and the 1 $\times 1$ bulk structure (CO-covered surface) is observed [38]. The oscillatory behavior of the reaction in a certain parameter regime [39] can be explained by this adsorbate-driven phase transition of the surface structure. Due to diffusion, $\mathrm{CO}$ is laterally mobile on the catalytic surface while oxygen diffusion can be neglected in the considered temperature range. Together with the above mentioned mechanisms, the interaction of lateral diffusivity and chemical reaction enables the emergence of a rich variety of complex concentration patterns on the platinum surface, ranging from reaction fronts and target patterns to spiral waves and chemical turbulence $[5,6]$.

For the present experiments, the $\operatorname{Pt}(110)$ single crystal sample was kept in a reaction chamber under ultrahigh vacuum conditions. The crystal was about $10 \mathrm{~mm}$ in diameter, and approximately $80 \%$ of the sample surface were covered by microlithographic decomposition with Ti. Since Ti is easily oxidized to $\mathrm{TiO}_{2}$, which is catalytically inert in the present reaction, only a small active fraction of the surface remained and thus effects of intrinsic global coupling through the gas phase [13] were minimized. Prior to each experiment, the crystal surface was prepared by repeated cycles of argon ion sputtering and annealing up to $750 \mathrm{~K}$. The catalyst temperature was controlled using a halogen projector lamp mounted directly behind the sample for heating of the crystal. Pressure gauges for $\mathrm{CO}$ and $\mathrm{O}_{2}$ allowed an accurate choice of the partial pressures of the reactants in the chamber. A photoemission electron microscope (PEEM) was used to monitor the surface of the catalyst sample $[40,41]$. Imaging the local work function across the surface, the PEEM yields spatially resolved information on the adsorbate coverage, since the local work function at a given point directly depends on the adsorbate coverage at this point. Different values of the local work function are then translated by the PEEM into different values of image brightness. The clean Pt surface has the lowest work function and, therefore, displays the brightest image. For the CO-covered surface, the work function is slightly increased $(\Delta \varphi=+0.3 \mathrm{eV})$, causing a small decrease in the intensity of the PEEM image compared to the clean surface. Finally, the O-covered surface appears nearly dark due to a more pronounced increase of the work function $(\Delta \varphi=+0.8 \mathrm{eV})$. We imaged a part of the surface of $500 \mu \mathrm{m}$ in diameter with a spatial resolution of about $1 \mu \mathrm{m}$. The temporal evolution was recorded with a frame rate of 25 images per second using a CCD camera.

In this setting, we implemented the TDAS feedback scheme devised by Pyragas [24] in the following way. We continuously recorded the integral intensity $I(t)$ of the PEEM image that has been normalized between zero for the oxygen covered surface and unity for the CO-covered surface. The integral intensity changed in the course of time due to pattern formation processes on the catalytic surface. From this quantity, the feedback signal was computed by taking the difference between the instantaneous integral image intensity and the intensity delayed by a chosen delay time $\tau$. This feedback signal, multiplied by an additional intensity factor $\mu$, was used as the input signal for the electronically operated dosing system for the $\mathrm{CO}$ gas. The $\mathrm{CO}$ partial pressure in the reaction chamber was thus modulated according to

$$
p_{\mathrm{CO}}(t)=p_{\mathrm{CO}}^{0}+\mu[I(t-\tau)-I(t)]
$$

In this way, a closed feedback loop was implemented, linking the dynamics of pattern formation on the sample surface with the partial pressure of $\mathrm{CO}$, one of the global control parameters of the system. Note that due to the finite pumping rate of the reaction chamber the variation of the $\mathrm{CO}$ partial pressure was not instantaneous but followed the feedback induced modulations with an additional delay $\tau_{i}$ of about half a second. In Sec. IV B, we investigate the effect of the control loop latency $\tau_{i}$ in numerical simulations using a standard realistic three-variable model of catalytic $\mathrm{CO}$ oxidation on $\operatorname{Pt}(110)$. For $\tau_{i} \ll \tau$ we did not find any significant qualitative difference to the results without control loop latency and, therefore, we neglect the effect of the intrinsic delay in the discussion of the results.

\section{EXPERIMENTAL RESULTS}

In this work, we study the dynamics of the catalytic $\mathrm{CO}$ oxidation on a $\mathrm{Pt}(110)$ single crystal surface under the effect of TDAS. We performed a series of experiments, varying both the feedback intensity $\mu$ and the delay time $\tau$ of the feedback scheme introduced in Eq. (1). The temperature and partial pressures of the reactants were chosen such that, in the absence of feedback, homogeneous oscillations were unstable and the system developed a state of highly irregular spiral-wave turbulence. A snapshot of this state is shown in Fig. 1(a). Note that the shape of concentration patterns on the $\mathrm{Pt}(110)$ surface is affected by anisotropy of surface diffusion of CO [42]. Diffusion of CO is faster in the [1 10$]$ direction than in the perpendicular [001] direction, causing an elongated, or, in the case of curved fragments, elliptical shape of the pattern.

When feedback is present, the system gradually synchronizes with increasing feedback intensity $\mu$. An example of the feedback-induced transition from turbulence to homogeneous oscillations is shown in Fig. 1. The upper part displays a sequence of three PEEM images. For zero feedback intensity, the system is in a state of fully developed spiral-wave turbulence [Fig. 1(a)]. There are no long living coherent patterns, and the turbulent state is characterized by small irregular rotating spiral-wave fragments that continuously move 


\section{B}

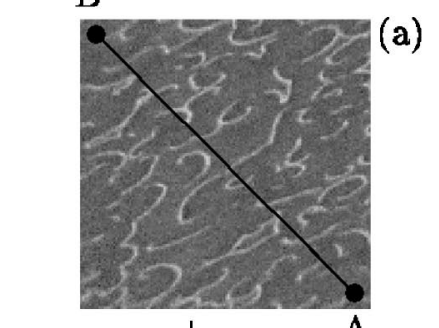

(a)

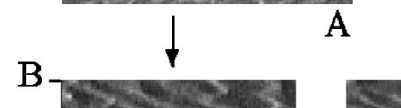

A

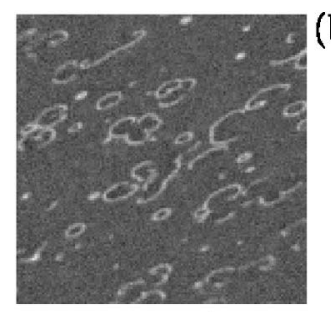

(b)

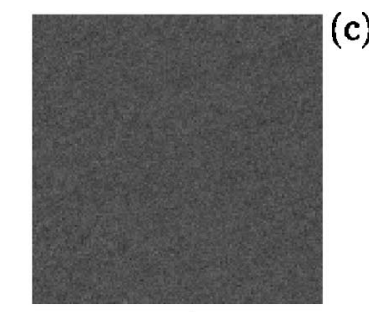

(c)

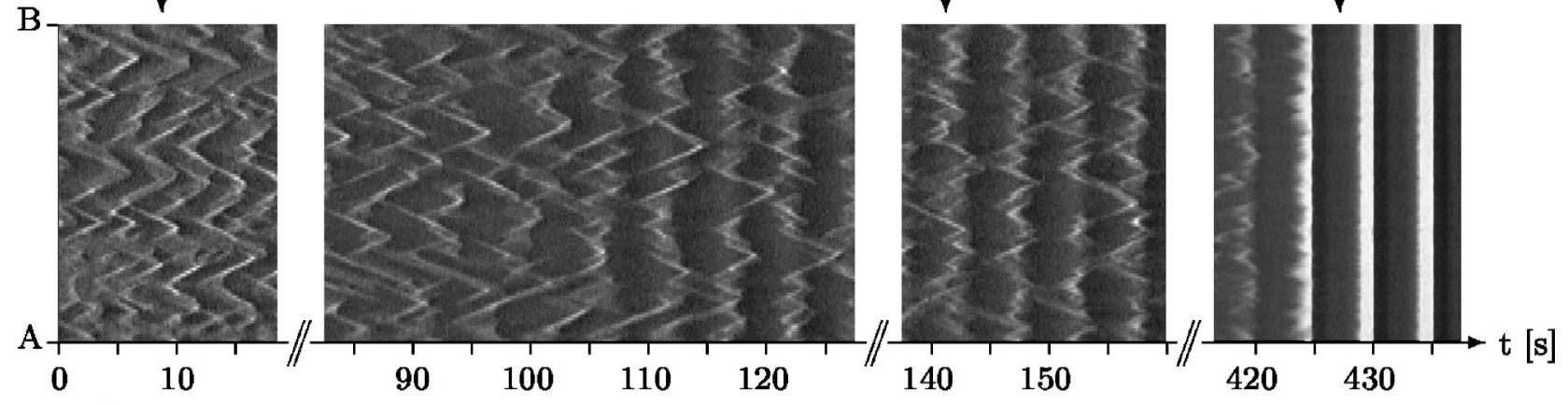

$\mu\left[10^{-5} \mathrm{mbar}\right]$

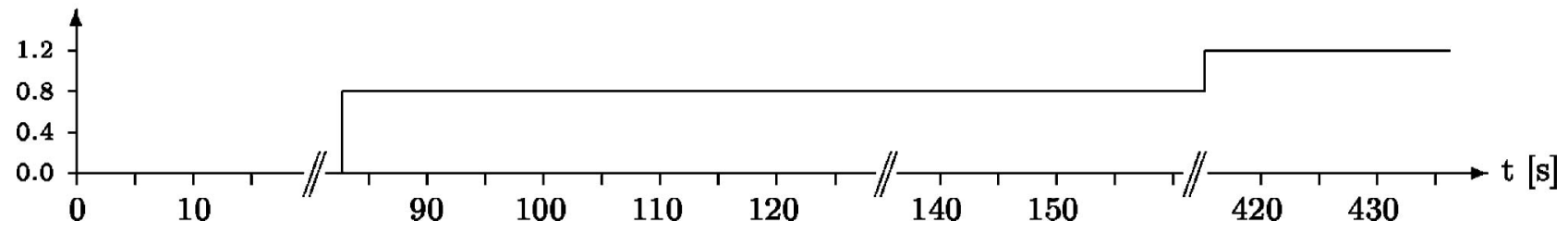

FIG. 1. Feedback-induced transition from chemical turbulence to homogeneous oscillations. Snapshots (top) of freely developing chemical turbulence in the absence of feedback (a), intermittent turbulence for small values of feedback intensity (b), and homogeneous oscillations for higher values of feedback intensity (c). Space-time plot (middle) for the transition from turbulence to homogeneous oscillations along the line $\mathrm{AB}$ indicated in image (a). From left to right, the plot shows, with increasing time, the turbulence for $\mu=0.0$, the transition to intermittent turbulence and the persistent state of intermittent turbulence for $\mu=0.77 \times 10^{-5} \mathrm{mbar}$, and the transition to homogeneous oscillations for $\mu=1.2 \times 10^{-5}$ mbar. Below the space-time diagram, the feedback intensity is plotted as a function of time. The parameters are $T=505 \mathrm{~K}, p_{\mathrm{O}_{2}}=4 \times 10^{-4} \mathrm{mbar}, p_{\mathrm{CO}}^{0}=9.5 \times 10^{-5} \mathrm{mbar}$, and $\tau=3 \mathrm{~s}$.

and break up. These waves emerge randomly and, upon meeting each other, undergo mutual annihilation. For intermediate values of $\mu$, oscillations are already synchronized to a certain extent and we can maintain a state of intermittent turbulence [Fig. 1(b)]. Intermittent turbulence has already been observed and characterized in the catalytic $\mathrm{CO}$ oxidation for a different kind of global delayed feedback [43], where two types of localized turbulent objects, ring-shaped bubble structures and spiral-wave fragments, were reported. They emerged in repeated reproduction cascades on a homogeneously oscillating background. In the experiment presented here, the intermittent turbulent state is dominated by localized, frequently breaking spiral fragments on an almost uniformly oscillating background. Bubblelike structures may also be observed. However, in most cases they burst and undergo breakups, again forming irregular spiral-wave fragments. Finally, for higher feedback intensities, complete synchronization is reached and the system performs homogeneous oscillations [Fig. 1(c)].

The space-time plot in the middle part of Fig. 1 further illustrates the process of synchronization. The plot is taken along the diagonal of the PEEM images from the top left to the bottom right hand corners, corresponding roughly to the
[001] direction along which surface diffusion of CO is slow. The feedback intensity $\mu$ is increased in the course of time (see the graph at the bottom of Fig. 1). The first part from the left $(0-18 \mathrm{~s})$ shows a space-time plot for the turbulent state of the system in the absence of feedback. The second part (from 82 s onwards) illustrates the transient from turbulence to intermittent turbulence and the third part (137-160 s) gives a space-time plot of the state of intermittent turbulence, where oscillations are already partly synchronized. Finally, after a short transient, we reach, in the fourth part, the completely synchronized state of homogeneous oscillations (416-437 s).

For synchronization of the system in dependence on the feedback intensity $\mu$, strong hysteresis effects are observed. In Fig. 2(a), we show the space-time diagram of an experiment where the feedback intensity is increased until homogeneous oscillations are reached and subsequently decreased again until synchronization is lost and patterns start to evolve. In this experiment, the parameters are chosen such that the initial state of the system in the absence of feedback is less turbulent, and shows evolving and interacting waves and large spirals. A snapshot of this state is displayed in Fig. 2(c). As shown in Fig. 2(b), the feedback intensity is in- 


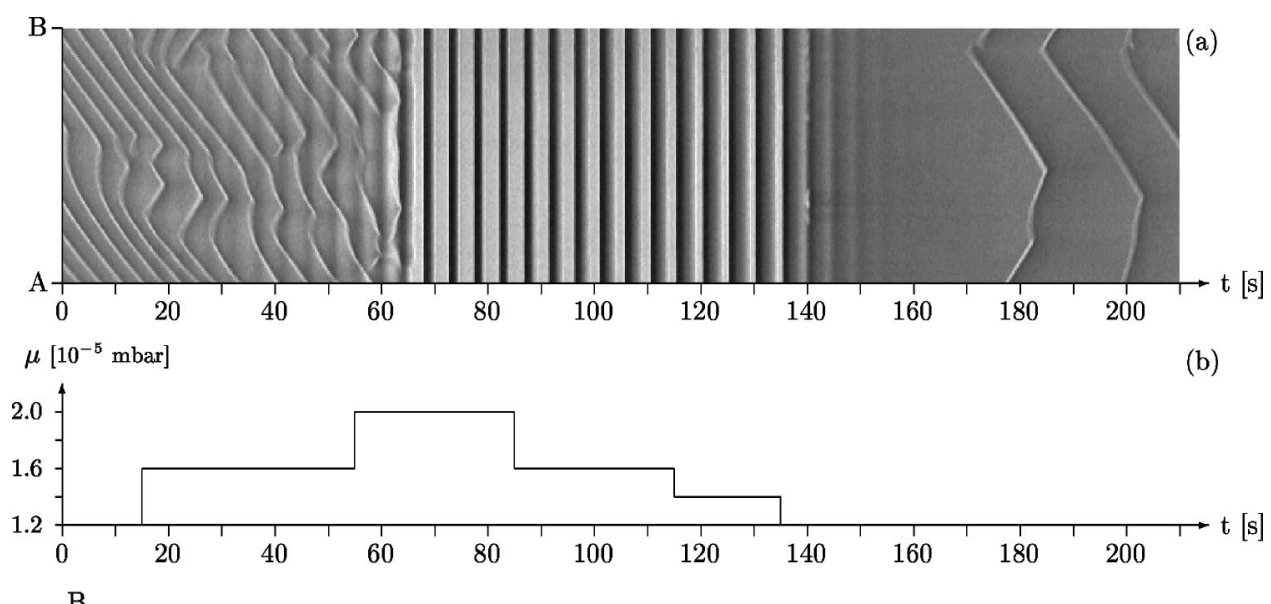

FIG. 2. Hysteresis for the transition from freely evolving patterns to complete synchronization. The space-time plot (a), taken along the line $A B$ indicated in image (c), shows the transition from uncontrolled pattern formation to homogeneous oscillations and the reverse transition from complete synchronization to freely evolving patterns. A plot of the corresponding feedback intensities as a function of time (b) is shown below the space-time diagram. Snapshot (c) is taken at low feedback intensity $(t=9 s)$. The parameters are $T=512 \mathrm{~K}, \quad p_{\mathrm{O}_{2}}=4 \times 10^{-4} \mathrm{mbar}$, $p_{\mathrm{CO}}^{0}=10.5 \times 10^{-5} \mathrm{mbar}$, and $\tau$ $=3.5 \mathrm{~s}$.

creased from $\mu=1.2 \times 10^{-5}$ to $2.0 \times 10^{-5} \mathrm{mbar}$, and then decreased again to $\mu=1.2 \times 10^{-5}$ mbar in the course of time. Obviously, coming from the uncontrolled state, the feedback intensity needed to synchronize the system is much higher than the feedback intensity necessary to maintain homogeneous oscillations when starting from the state of complete synchronization and decreasing the feedback intensity. When synchronization is lost at a low feedback intensity, the wavelength of the patterns which start to evolve is, at first, much longer than it was at the beginning of the experiment. However, it decreases with time and soon the system reaches its initial state again (not shown in Fig. 2).

In the state of control, the period $T$ of homogeneous oscillations depends on the choice of the delay time $\tau$ in the

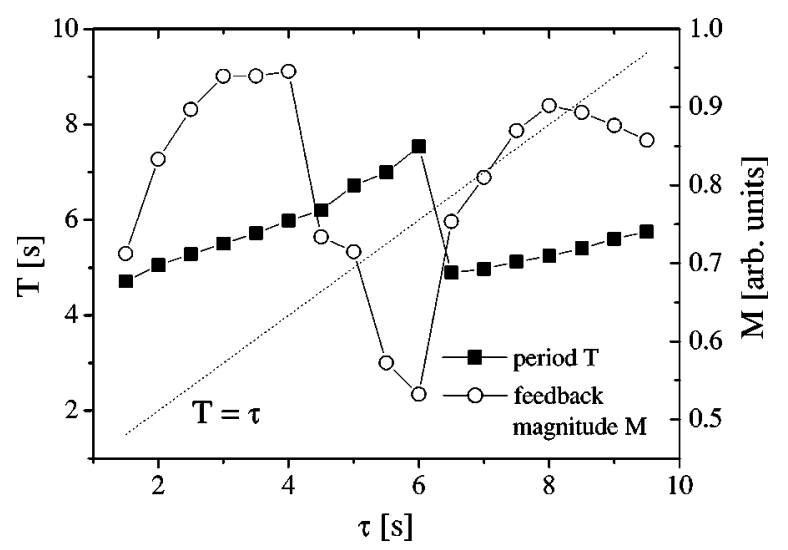

FIG. 3. Period $T$ of homogeneous oscillations (black squares) and feedback magnitude $M$ (open circles) in dependence on the delay time $\tau$. The parameters are temperature, partial pressure of oxygen, base partial pressure of $\mathrm{CO}$, and feedback intensity, respectively: $T=515 \mathrm{~K}, p_{\mathrm{O}_{2}}=4 \times 10^{-4} \mathrm{mbar}, p_{\mathrm{CO}}^{0}=10.0 \times 10^{-5} \mathrm{mbar}$, and $\mu / p_{\mathrm{CO}}^{0}=0.32$. feedback scheme. In Fig. 3, the period $T$ is plotted versus the delay time $\tau$ (black squares). A series of experiments was performed to obtain this plot. At the beginning of each experiment, the $\mathrm{CO}$ supply was kept closed and only oxygen was present in the reaction chamber, causing a uniform oxygen coverage on the sample surface. At $t=0$, the $\mathrm{CO}$ valve was opened to its predefined value. The parameters were chosen such that, in the absence of feedback, chemical turbulence would spontaneously develop. In these experiments, however, the feedback intensity factor $\mu$ was chosen large enough to prevent the emergence of turbulence and to stabilize homogeneous oscillations. The oscillation period was then determined for different values of the delay time $\tau$.

Superposed in the same plot, we show the magnitude of the feedback signal that acts on the system at different delay times. To this end, the quantity $M=\langle|I(t-\tau)-I(t)|\rangle$ is plotted, which is the time average over the modulus of the feedback signal (open circles in Fig. 3). We call $M$ the feedback magnitude. Since the feedback signal is oscillating around zero, we compute the feedback magnitude as the time average of the modulus of the feedback signal. Thus, this quantity is a measure for the absolute average driving force that is imposed on the system by the feedback and hence quantifies the invasiveness of the feedback. Finally, we added to the plot the line given by $T=\tau$, representing all points for which the optimal case of a vanishing feedback signal would be reached.

The experimental result shows clearly that, as with increasing delay time the values for $T$ approach the line where $T$ equals $\tau$, the magnitude of the feedback signal is decreased by about a factor of two. Hence, we were able to reduce the invasiveness of the feedback considerably by optimizing the choice of the delay time in the feedback scheme.

On the other hand, the feedback signal $F(t)=\mu[I(t-\tau)$ $-I(t)]$ should become vanishingly small at $\tau=T$ if uniform 
oscillations with period $T$ were stabilized by a noninvasive feedback. However, we could not reach this optimal case in our experiments. Instead, a jump always occurred around the line given by $T=\tau$, and the system evaded the point where its oscillation period would have become equal to the delay time. To understand the origin of such a behavior, theoretical investigations of the global TDAS scheme have been performed.

\section{THEORETICAL INVESTIGATIONS}

\section{A. Phase dynamics equation}

Some properties of a spatially extended system under the influence of TDAS can already be understood in terms of a simple analysis for the uniform system. For a limit-cycle oscillator, the orbital shape, amplitude, and frequency of oscillations are well-defined characteristic features. If we apply a small perturbation, the original orbital form and amplitude will be reestablished after some relaxation time. The phase, however, will not recover its initial value but will maintain a small shift induced by the perturbation. By an adiabatic elimination of the amplitude variable, the description of oscillatory dynamics may be reduced to a simple equation for the phase variable $\phi$. The phase dynamics equation of a single oscillator under the effect of weak TDAS should take the form (see Ref. [44] and the Appendix)

$$
\dot{\phi}=\omega+\mu f[\phi(t)-\phi(t-\tau)] .
$$

Here, $\omega$ is the oscillation frequency in absence of feedback, $\tau$ is the delay time, and $\mu$ is the coefficient characterizing the feedback intensity (note that the phase equation is only valid for $\mu \ll 1)$. Generally, the function $f[\phi(t)-\phi(t-\tau)]$ is $2 \pi$ periodic and satisfies the conditions $f(0)=f(2 \pi)=0$. For a harmonic oscillator close to the onset of oscillations, described by the Stuart-Landau equation [45], this function is given by (see the Appendix)

$$
f(\Delta \phi)=a \sin (\Delta \phi)+b \cos (\Delta \phi)-b,
$$

with $\Delta \phi=\phi(t)-\phi(t-\tau)$, where $a$ and $b$ are fixed parameters, expressed as $a=\cos \chi+\beta \sin \chi$ and $b=\sin \chi-\beta \cos \chi$ in terms of the coefficients $\beta$ and $\chi$ of the Stuart-Landau equation with TDAS.

Harmonic oscillations with $\phi(t)=\Omega t$ are special solutions of the phase equation (2), and their oscillation frequency $\dot{\phi}=\Omega$ should satisfy

$$
\Omega=\omega+\mu f(\Omega \tau)
$$

The solutions of this equation can be constructed by writing it as

$$
\mu=\frac{\Omega-\omega}{f(\Omega \tau)} .
$$

For each oscillation frequency $\Omega$, it determines the respective value of the feedback intensity $\mu$. Figure 4 shows thus (a)

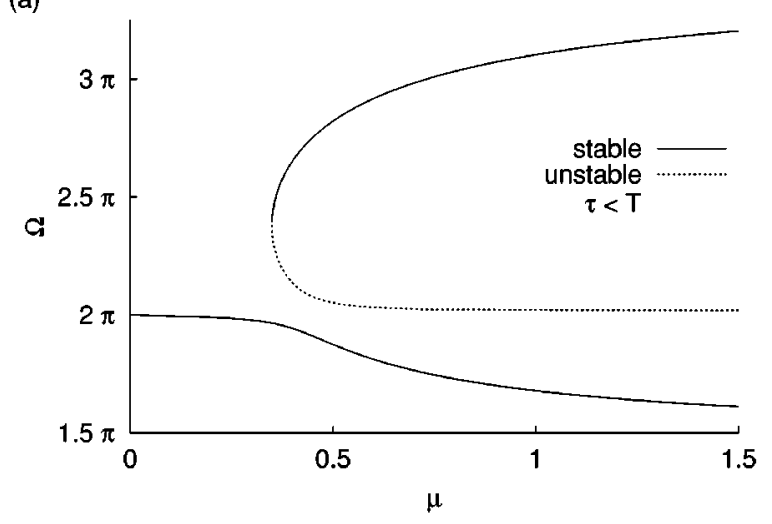

(b)

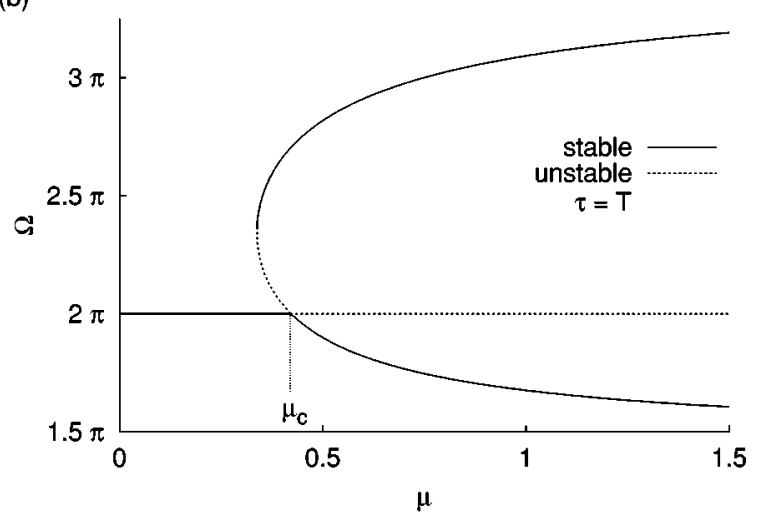

(c)

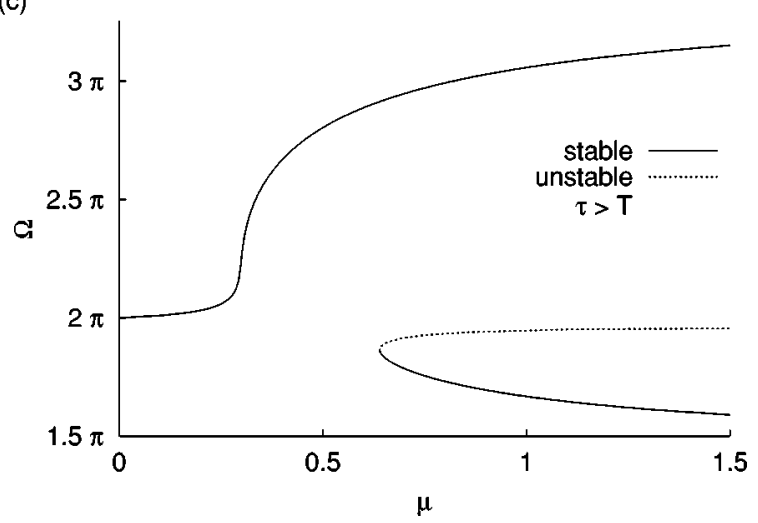

FIG. 4. Oscillation frequency as a function of the feedback intensity for three different delay times (a) $\tau=0.985$, (b) $\tau=1$, and (c) $\tau=1.015$. The parameters are $\omega=2 \pi, \beta=3$, and $\chi=\pi / 6$. The transcritical bifurcation takes place at $\mu_{c} \approx 0.42$. Dotted lines show unstable branches.

obtained solutions for three different delay times, choosing as an example the function $f$ given by Eq. (3) for $\beta=3$ and $\chi=\pi / 6$.

When the delay time is equal to the natural oscillation period, $\tau=T_{0}=2 \pi / \omega$, Eq. (4) always has a solution $\Omega$ $\neq \omega$ with vanishing feedback signal. However, other solutions are additionally present at sufficiently high feedback intensities, as seen from Fig. 4(b). For these additional solutions, $\Omega=\omega$ and the feedback signal is not vanishing. They emerge via a transcritical bifurcation at $\mu=\mu_{c}$. Generally, the bifurcation point is given by 


$$
\mu_{c}=\lim _{\Omega \rightarrow \omega} \frac{\Omega-\omega}{f\left(2 \pi \frac{\Omega}{\omega}\right)}=\frac{\omega}{2 \pi f^{\prime}(0)}
$$

When $\tau \neq T$, the transcritical bifurcation is replaced by a saddle-node bifurcation, leading to the appearance of two new solution branches at sufficiently high feedback intensities, as shown in Figs. 4(a) and 4(c).

The phase dynamics equation (2) can further be used to analyze the stability of various solution branches. We apply small perturbations to the phase, $\phi=\Omega t+\delta \phi$, and, after linearization, obtain an equation for $\delta \phi$ :

$$
\dot{\delta \phi}=\mu f^{\prime}(\Omega \tau)[\delta \phi(t)-\delta \phi(t-\tau)]
$$

With an ansatz $\delta \phi(t)=e^{\lambda t / \tau}$, we find

$$
\lambda=q\left(1-e^{-\lambda}\right),
$$

where $q=\tau \mu f^{\prime}(\Omega \tau)$. Thus, the solution with frequency $\Omega$ is stable, if $\operatorname{Re} \lambda<0$.

Suppose that $\tau=T_{0}$ and investigate the stability of the solution with $\Omega=\omega$. In this case, the coefficient $q$ can be written as

$$
q=\frac{\mu}{\mu_{c}}
$$

by taking into account Eq. (6) and $f^{\prime}(\Omega \tau)=f^{\prime}(2 \pi)$ $=f^{\prime}(0)$. By analyzing the roots of the characteristic equation (8), it can be shown that the solution with $\Omega=\omega$ becomes unstable at the transcritical bifurcation point $\mu=\mu_{c}$. A similar analysis can be performed for other solution branches and also for $\tau \neq T$. As a result, we find that only the solution branches, indicated by solid lines in Fig. 4, are stable. Thus, our study, based on the general phase dynamics equation, predicts that at $\tau=T_{0}$ the solution with $\Omega=\omega$ should become unstable at sufficiently high feedback intensities and will be replaced by one of the two possible solutions with $\Omega \neq \omega$.

We performed a series of numerical simulations of Eq. (2) tracing the oscillation period $T=2 \pi / \Omega$ as a function of the delay time $\tau$. Figure 5(a) displays the dependence of $T$ on the delay time $\tau$ for a feedback intensity below the critical value (for $\mu<\mu_{c}$ ). We see that at $\tau=T_{0}$ the solution with $\Omega=\omega$ is indeed realized. However, if the feedback intensity is increased above its critical value $\mu_{c}$, the system cannot reach such state with a vanishing control signal and a hysteresis effect takes place, as shown in Fig. 5(b).

Comparing Fig. 5 with Fig. 3, we note that the behavior of the oscillation period under varying the delay time is qualitatively similar in the experiment and in our numerical simulations based on the phase dynamics equation with $\mu>\mu_{c}$. We should, however, remember that the phase equation is, strictly speaking, valid only for weak feedbacks. Therefore, to analyze the system behavior at stronger feedbacks, additional simulations based on the realistic reaction model are needed.
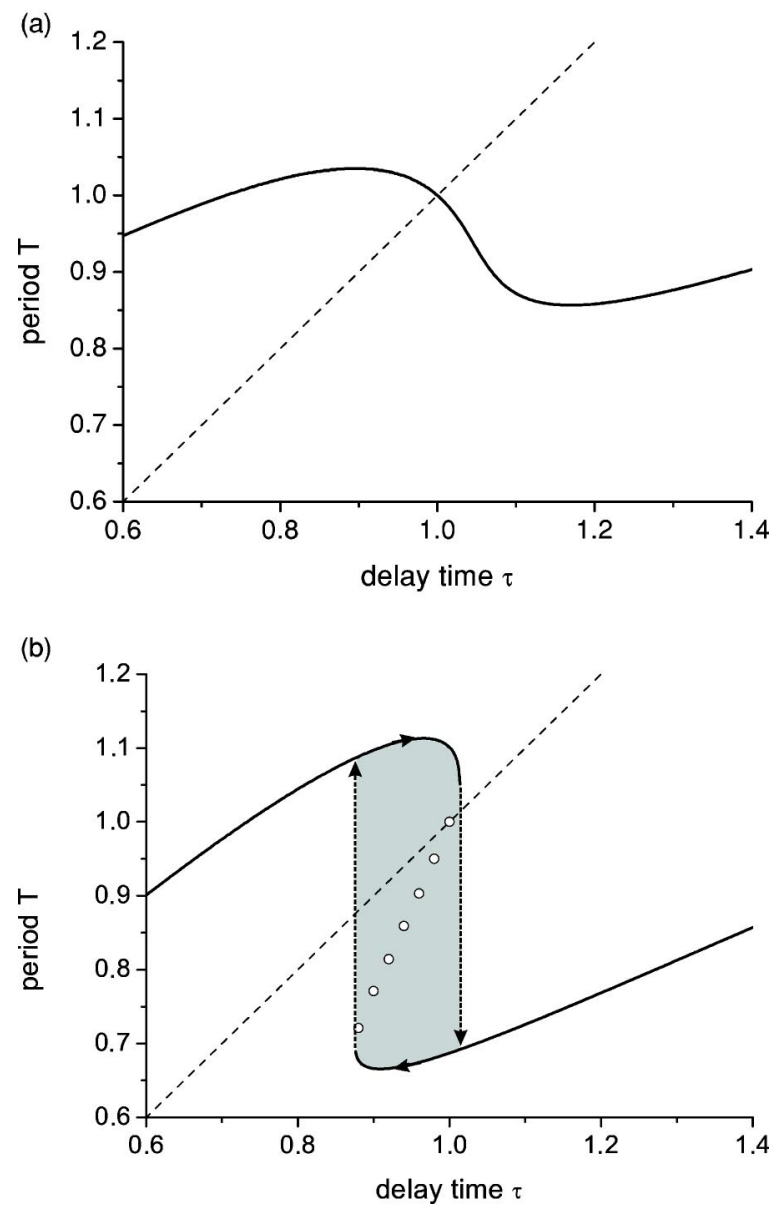

FIG. 5. Oscillation period as function of delay time obtained from numerical simulations of the phase dynamics equation for a feedback intensity (a) of $\mu=0.2$ and (b) of $\mu=0.6$. The parameters are as in Fig. 4. Hysteresis effects are found in the gray shaded region in (b). Open circles indicate unstable solutions yielded by Eq. (4).

\section{B. Realistic reaction model}

Catalytic CO oxidation on $\mathrm{Pt}(110)$ single crystal surfaces is described by a model $[9,8,11]$ that consists of three coupled ordinary differential equations

$$
\begin{aligned}
& \dot{u}=k_{1} s_{\mathrm{CO}} p_{\mathrm{CO}}\left(1-u^{3}\right)-k_{2} u-k_{3} u v, \\
& \dot{v}=k_{4} p_{\mathrm{O}_{2}}\left[s_{\mathrm{O}, 1 \times 1}+s_{\mathrm{O}, 1 \times 2}(1-w)\right](1-u-v)^{2}-k_{3} u v, \\
& \dot{w}=k_{5}\left(\frac{1}{1+\exp \left(\frac{u_{0}-u}{\delta u}\right)}-w\right),
\end{aligned}
$$

where the variables $u, v$, and $w$ represent the CO coverage, the oxygen coverage, and the fraction of the surface found in the nonreconstructed $1 \times 1$ phase, respectively. The model takes into account the adsorption of $\mathrm{CO}$ and oxygen, the reaction between the two adsorbed species, the desorption of $\mathrm{CO}$, and the adsorbate-dependent structural change of the 

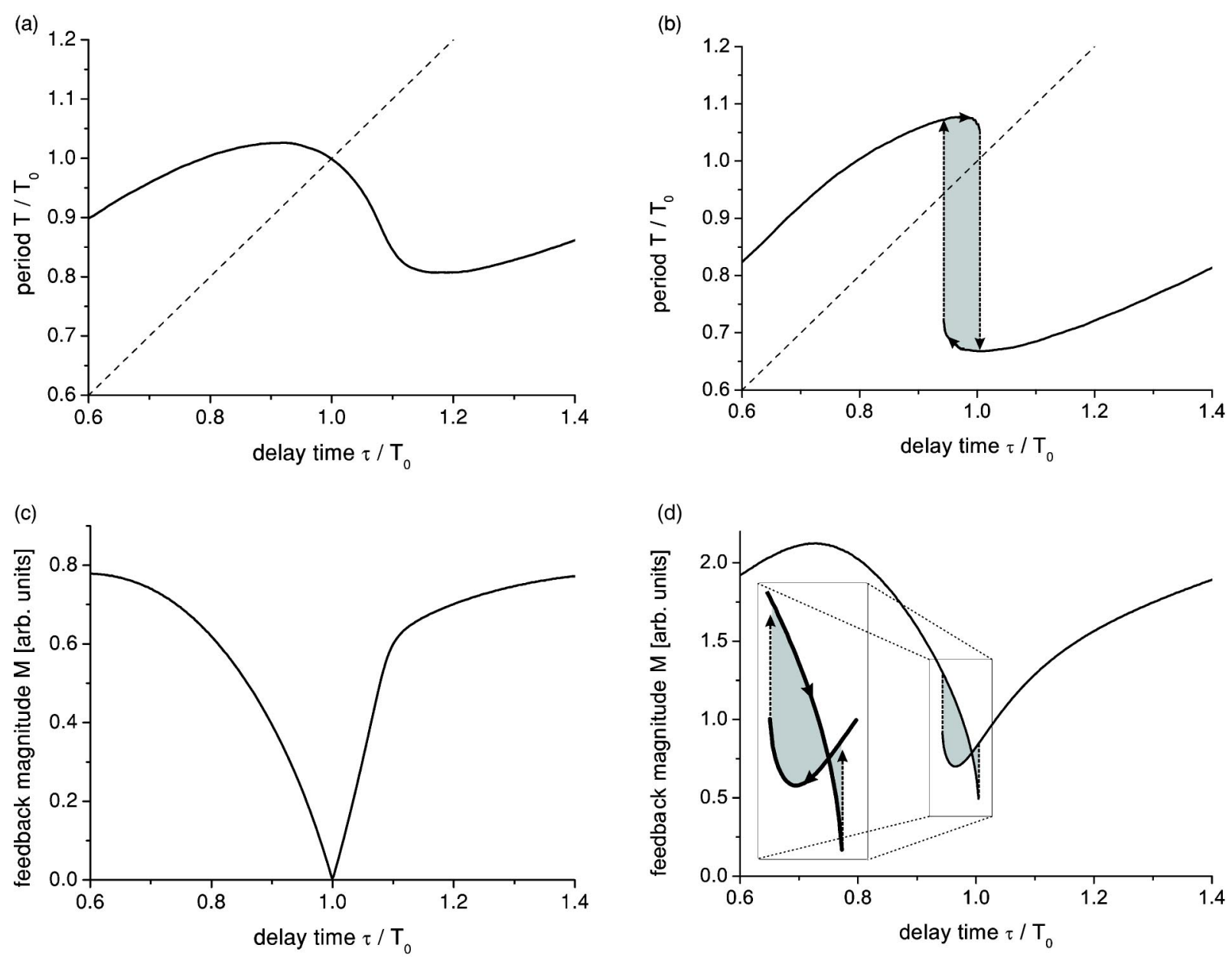

FIG. 6. Oscillation period [(a) and (b)] and feedback magnitude [(c) and (d)] as functions of the delay time obtained from numerical simulations of the realistic model of $\mathrm{CO}$ oxidation on $\mathrm{Pt}(110)$ for two different feedback intensities $[(\mathrm{a})$ and $(\mathrm{c})] \mu / p_{\mathrm{CO}}^{0}=0.01$ and $[(\mathrm{b})$ and (d) $] \mu / p_{\mathrm{CO}}^{0}=0.03$. The model parameters are $k_{1}=3.14 \times 10^{5} \mathrm{~s}^{-1} \mathrm{mbar}^{-1}, k_{2}=10.21 \mathrm{~s}^{-1}, k_{3}=283.8 \mathrm{~s}^{-1}, k_{4}=5.86 \times 10^{5} \mathrm{~s}^{-1} \mathrm{mbar}^{-1}, k_{5}$ $=1.61 \mathrm{~s}^{-1}, s_{\mathrm{CO}}=1.0, s_{\mathrm{O}, 1 \times 1}=0.6, s_{\mathrm{O}, 1 \times 2}=0.4, u_{0}=0.35, \delta u=0.05, p_{\mathrm{CO}}^{0}=4.82 \times 10^{-5} \mathrm{mbar}$, and $p_{\mathrm{O}_{2}}=13.1 \times 10^{-5} \mathrm{mbar}$.

$\operatorname{Pt}(110)$ surface. Some processes, such as the formation of subsurface oxygen or roughening of the catalytic surface, are neglected here.

The TDAS feedback scheme should be further included into the model. For simplicity, we introduce it via a dependence of the $\mathrm{CO}$ partial pressure on the $\mathrm{CO}$ coverage,

$$
p_{\mathrm{CO}}(t)=p_{\mathrm{CO}}^{0}+\mu[u(t-\tau)-u(t)]
$$

where $p_{\mathrm{CO}}^{0}$ is the base $\mathrm{CO}$ partial pressure, $\mu$ is the feedback intensity, and $\tau$ is the delay time. In the experiments, the feedback signal was generated using the integral PEEM intensity $I$, which nonlinearly depends on both the $\mathrm{CO}$ and the oxygen coverages.

Numerical simulations of the model (10)-(13) were performed using a set of parameter values for which the medium is in the oscillatory state and, once the mobility of adsorbed $\mathrm{CO}$ molecules is taken into account, diffusioninduced turbulence spontaneously develops [34]. The period of oscillations in the absence of feedback was $T_{0}=2.44 \mathrm{~s}$.

Figure 6 shows the results of numerical simulations of the realistic model. Here, the dependences of the oscillation period $T$ and of the feedback signal magnitude $M=\langle| u(t-\tau)$ $-u(t)|\rangle$ on the delay time $\tau$ are displayed. For relatively weak feedbacks [Fig. 6(a) and 6(c)], a state with vanishing feedback signal $M$ is realized at $\tau=T_{0}$. When the feedback is increased, this state, however, becomes unstable and cannot be reached in our simulations. Instead, a hysteresis effect is observed [Fig. 6(b)]. If we start with a short delay time $\tau$ and increase it, the oscillation period $T$ is first larger than $T_{0}$ and gradually grows. When the delay time becomes slightly larger than $T_{0}$, the oscillation period $T$ abruptly jumps down to a value below $T_{0}$ and then slowly increases. If we move in the opposite direction and gradually decrease the delay time, a jump to the upper branch occurs slightly below $\tau=T_{0}$. Though the feedback signal is decreased close to $\tau=T_{0}$, it does not drop down to zero at this point and a hysteresis effect for this property is also observed [Fig. 6(d)].

In Sec. II, we explained that for technical reasons the feedback signal, applied to the system in the experiment, took effect with an additional intrinsic time delay $\tau_{i}$. It is known from both experimental [46] and theoretical [47] studies that a control loop latency in the application of TDAS might effect the size of the domain of control of the system. Here, however, we are not investigating the size of the domain of control but we are concerned with qualitatively ex- 

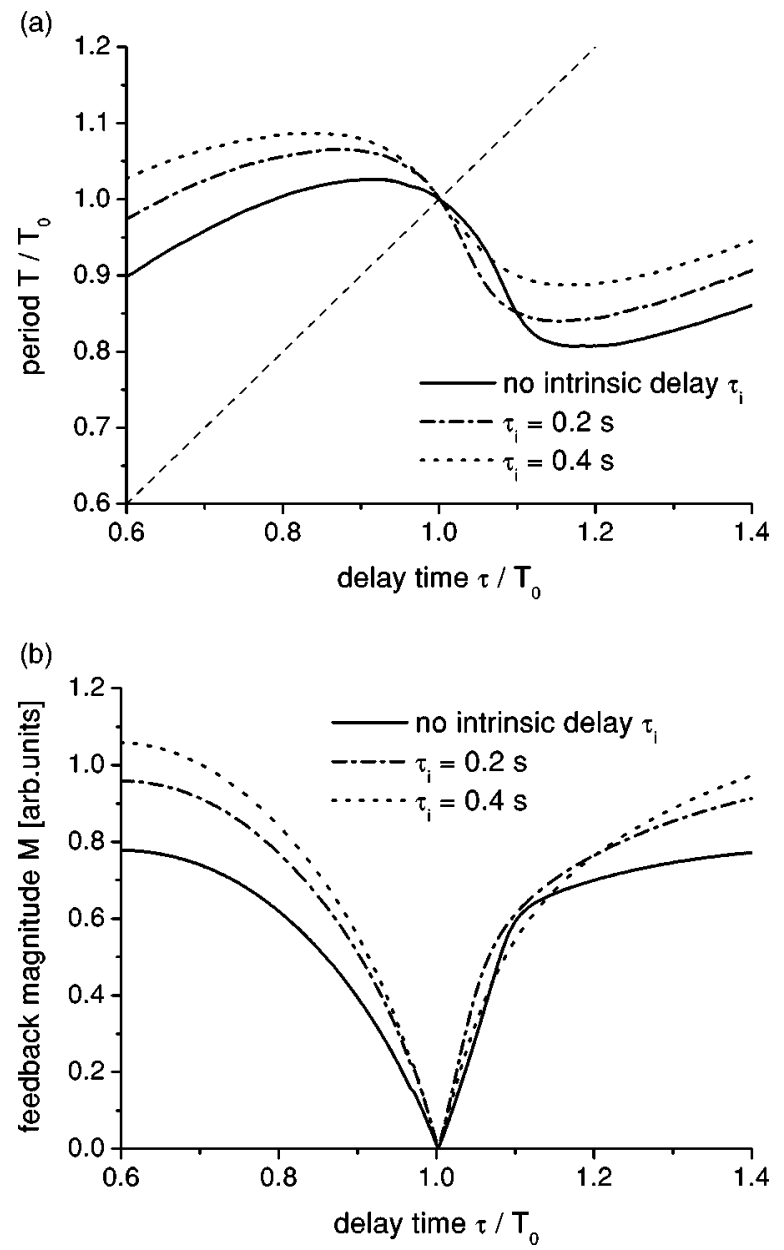

FIG. 7. Effects of control loop latency: oscillation period (a) and feedback magnitude (b) as functions of delay time for different values of intrinsic delay. The model parameters are as in Figs. 6(a) and 6(c).

plaining the behavior of the system in the state of control for varying delay time. In order to study the impact of control loop latency on our results, we repeated the computations that led to the results shown in Figs. 6(a) and 6(c). This time, the intrinsic delay $\tau_{i}$, which is due to the finite pumping rate of the reaction chamber, was included by extending the model (10)-(12) with an additional equation for the time evolution of the $\mathrm{CO}$ partial pressure:

$$
\dot{p}_{\mathrm{CO}}=-\frac{1}{\tau_{i}}\left\{p_{\mathrm{CO}}-p_{\mathrm{CO}}^{0}-\mu[u(t-\tau)-u(t)]\right\} .
$$

Figure 7 shows the oscillation period $T$ (a) and the feedback magnitude $M(\mathrm{~b})$ as a function of delay time $\tau$ for two different values of the intrinsic delay $\tau_{i}$ in comparison to the results for zero intrinsic delay which are, of course, identical to Figs. 6(a) and 6(c). With an increasing intrinsic delay, the curve describing the dependence of the oscillation period on the delay time experiences a shift to higher values but maintains its original shape. The change in the feedback magnitude, on the other hand, consists roughly in an increase by some multiplying factor. However, both the oscillation period and the feedback magnitude maintain qualitatively simi- lar shapes in their dependence on $\tau$ for vanishing $\tau_{i}$ and different nonzero $\tau_{i}$. In particular, we point out that the effect of control loop latency does not simply amount to adding up the intrinsic delay $\tau_{i}$ and the delay $\tau$ from the control scheme. Instead, the intersection point of the curve giving the dependence of $T$ on $\tau$ with the line for which $T=\tau$ is not shifted when control loop latency is introduced and, as a consequence, the minimum of the feedback magnitude remains in the same position as well. The effect of an intrinsic delay gets less pronounced when approaching the point for which $T=\tau$ which is of particular interest for our present investigation.

\section{Spatially extended systems}

Diffusion plays a principal role in the considered system, since it destabilizes the state of uniform oscillations and brings up chemical turbulence [34]. While individual oscillators show regular periodic dynamics, their population displays diffusion-induced spatiotemporal chaos. Diffusion terms were not, however, taken into account in the phase dynamics equation (2) and in the realistic model (10)-(13) of the $\mathrm{CO}$ oxidation reaction. Therefore, these equations describe only the effect of TDAS on the uniform oscillation mode.

In the absence of feeback, the uniform oscillatory mode cannot suppress the growth of other spatial modes, and turbulence develops. When global feedback is applied, only the uniform spatial mode can be directly influenced by the controlling force. The other spatial modes are affected by such feedback because they are coupled to the uniform mode. In other words, they "feel" the presence of a feedback only to the extent that the dynamics of the uniform mode is modified.

If the delay time is equal to the oscillation period $(\tau$ $\left.=T_{0}\right)$ and the feedback signal is vanishing $(M=0)$, the periodic uniform mode would not be modified by the feedback. Moreover, its interactions with nonuniform spatial modes would not be modified either. But then these other spatial modes should behave exactly in the same way as in the absence of feedback, so that spatiotemporal chaos would develop.

These simple general arguments indicate that a noninvasive suppression of diffusion-induced chemical turbulence by global feedbacks is not possible. When the TDAS method is applied, a stabilization of periodic uniform oscillations with $\Omega=\omega$ cannot be realized at $\tau=T_{0}$. Though the phase dynamics equation (2) and the realistic model (10)-(13) without diffusion predict that the state with $\Omega=\omega$ is stable for sufficiently weak feedbacks with $\mu<\mu_{c}$, such a state would always be unstable when diffusion is included and interactions with nonuniform spatial modes are allowed. Note that the above arguments do not exclude the possibility that a nonuniform oscillatory pattern becomes stabilized as a result of the global feedback [30].

\section{CONCLUSIONS}

We studied the control of chemical turbulence in the catalytic CO oxidation on a $\operatorname{Pt}(110)$ single crystal surface by 
means of time-delay autosynchronization (TDAS). It was found that synchronization can be gradually induced in the system: with increasing feedback intensity a transition from chemical turbulence to homogeneous oscillations takes place via a state of intermittent turbulence. For the transition from the uncontrolled state to homogeneous oscillations, significant hysteresis effects were observed. Varying the delay time, we were able to lower the magnitude of the feedback signal by a factor of $50 \%$, thus reducing the invasiveness of the feedback considerably. However, we could not reach the ideal case of a vanishingly small feedback signal in the state of control since TDAS does not allow one to directly target specific points in phase space for arbitrary feedback intensities. Instead, we found that the oscillation period $T$ as a function of the delay time $\tau$ displays a discontinuity around the point where $T=\tau$. We thus conjectured that the point for which $T=\tau$ was unstable in the experiment.

To understand the dynamics of the system under the effect of TDAS, a phase dynamics equation for a single oscillator in the presence of TDAS has been derived. From the phase equation, we obtained a bifurcation diagram for the oscillation frequency of the system with the feedback intensity as bifurcation parameter. A linear stability analysis showed that for feedback intensities below a certain threshold, the solution with vanishing feedback signal is stable, while at the threshold a transcritical bifurcation occurs and this solution becomes unstable. Simple general arguments have been presented to show that, once diffusion is included, it would make stable uniform oscillations with $T=\tau$ impossible.

In numerical simulations of both the phase dynamics approximation and a realistic three-variable model for $\mathrm{CO}$ oxidation on $\mathrm{Pt}(110)$, the experimental results could be comfirmed and the occurence of a discontinuity in the dependence of $T$ on $\tau$ could be reproduced for feedback intensities above the critical threshold. The hysteresis effects in the dependence of $T$ on $\tau$ could not, however, be checked in the present experiments because, for technical reasons, we were not able to vary the delay time within the same measurement, and a separate experiment had to be carried out for each new value of the delay time.

\section{ACKNOWLEDGMENTS}

We gratefully acknowledge financial support of the Deutsche Forschungsgemeinschaft (Sonderforschungsbereich 555 "Komplexe Nichtlineare Prozesse"). We thank E. Schöll for fruitful discussions and M. Pollmann for his help concerning the experimental setting. We are grateful to I.G. Kevrekidis for providing the microlithographically fabricated crystal.

\section{APPENDIX: DERIVATION OF A PHASE EQUATION IN THE PRESENCE OF TDAS}

The specific properties of a limit cycle oscillator may differ strongly from one system to another. However, sufficiently close to the onset of oscillations, the behavior of any such system takes a universal form. Close to a bifurcation point, the dynamics of the system is governed by only criti- cal modes which evolve on significantly longer time scales than the other noncritical modes. The dynamics of these remaining modes is "enslaved" to the evolution of the critical modes so that they may be eliminated adiabatically. As a consequence, the system of ordinary differential equations that describes the specific system reduces to a simple universal equation for the complex oscillation amplitude $\eta(t)$, which is known as the Stuart-Landau equation. In order to study the behavior of an oscillator in the presence of TDAS, we extend the Stuard-Landau equation by an additional feedback term $F(t)$, thus obtaining

$$
\dot{\eta}=\left(1-i \omega_{0}\right) \eta-(1+i \beta)|\eta|^{2} \eta+F(t),
$$

with

$$
F(t)=\mu e^{i \chi}[\eta(t)-\eta(t-\tau)] .
$$

Here, $\omega_{0}$ is the frequency of small-amplitude oscillations, the coefficient $\beta$ determines the nonlinear frequency shift, and the parameter $\chi$ accounts for a possible phase shift in the application of the control force. The feedback intensity is specified by the coefficient $\mu$. We assume that the feedback is weak, $\mu \ll 1$.

The Stuart-Landau equation (A1) is written in a dimensionless form, by choosing as a time unit the relaxation time which is proportional to the distance from the bifurcation point. Since this relaxation time diverges at the Hopf bifurcation, whereas the oscillation period remains finite, we have $\omega_{0} \gg 1$ in the neighborhood of the Hopf bifurcation.

Through the ansatz $\eta(t)=\rho(t) e^{-i \phi(t)}$ for the complex oscillation amplitude $\eta$, the oscillation phase $\phi$ is introduced and the feedback term is written as

$$
F(t)=\mu e^{i \chi}\left[\rho(t) e^{-i \phi(t)}-\rho(t-\tau) e^{-i \phi(t-\tau)}\right] .
$$

Substituting Eq. (A3) into Eq. (A1) we obtain, by separating real and imaginary parts, the following equations for the real amplitude $\rho(t)$ and for the phase $\phi(t)$, respectively:

$$
\begin{aligned}
& \dot{\rho}=\rho\left(1-\rho^{2}\right)+\mu[\rho(t) \cos \chi-\rho(t-\tau) \cos (\chi+\Delta \phi)], \\
& \dot{\phi}=\omega_{0}+\beta \rho^{2}-\mu\left[\sin \chi-\frac{\rho(t-\tau)}{\rho(t)} \sin (\chi+\Delta \phi)\right],
\end{aligned}
$$

where $\Delta \phi(t)=\phi(t)-\phi(t-\tau)$.

Since the feedback is weak $(\mu \ll 1)$, it leads to only small variations of the real amplitude $\rho$, that is, we have $\rho(t)=1$ $+\delta \rho(t)$ where $\delta \rho$ is of order $\mu$. Therefore, Eq. (A4) approximately yields

$$
\dot{\delta \rho}=-2 \delta \rho+\mu[\cos \chi-\cos (\chi+\Delta \phi)] .
$$

Thus, small perturbations of $\rho$ adjust to the variation of $\Delta \phi$ within the relaxation time of order unity. On the other hand, the variations of $\Delta \phi$ are slow and are characterized by time scales of order $1 / \mu$ which are much larger than unity. Hence, the adiabatic approximation can be used and we obtain

$$
\delta \rho=\frac{\mu}{2}[\cos \chi-\cos (\chi+\Delta \phi)] .
$$


Substituting this result into Eq. (A5) and again retaining only the terms linear in $\mu$, the following approximate phase dynamics equation is derived:

$$
\dot{\phi}=\omega+\mu f(\Delta \phi) .
$$

The function $f(\Delta \phi)$ in this equation is given by

$$
f(\Delta \phi)=a \sin (\Delta \phi)+b \cos (\Delta \phi)-b,
$$

and the notations $\omega=\omega_{0}+\beta, \quad a=\cos \chi+\beta \sin \chi$, and $b$ $=\sin \chi-\beta \cos \chi$ are introduced.

Now we can check that the variations of $\Delta \phi$ are indeed slow. We define the slow phase variable $\varphi(t)=\phi(t)-\omega t$, note that $\Delta \phi=\Delta \varphi+\omega \tau$, and write Eq. (A8) as

$$
\dot{\varphi}=\mu f(\Delta \varphi+\omega \tau) .
$$

Thus, the rate of variation of $\varphi$ is proportional to the feedback intensity and is small, $\mu \ll 1$. Because $\Delta \phi$ differs from $\Delta \varphi$ only by a constant, the same is true for $\Delta \phi$.

Though we have derived the phase dynamics equation (A8) in this appendix only starting from the Stuart-Landau equation, a similar equation can be found for any limitcycle oscillator under the action of sufficiently weak TDAS. Generally, the $2 \pi$-periodic function $f(\Delta \phi)$ would have a form different from (A9) satisfying the condition $f(0)$ $=0$.
[1] A. S. Mikhailov, Foundations of Synergetics I \& II (Springer, Berlin, 1994).

[2] A. Winfree, Science 175, 634 (1972).

[3] A. Zaikin and A. Zhabotinsky, Nature (London) 225, 535 (1970).

[4] Oscillations and Travelling Waves in Chemical Systems, edited by R. Fields and M. Burger (Wiley, New York, 1985).

[5] R. Imbihl and G. Ertl, Chem. Rev. 95, 697 (1995).

[6] S. Jakubith, H. H. Rotermund, W. Engel, A. v. Oertzen, and G. Ertl, Phys. Rev. Lett. 65, 3013 (1990).

[7] T. Engel and G. Ertl, Adv. Catal. 28, 1 (1979).

[8] M. Bär, M. Eiswirth, H. Rotermund, and G. Ertl, Phys. Rev. Lett. 69, 945 (1992).

[9] K. Krischer, M. Eiswirth, and G. Ertl, J. Chem. Phys. 96, 9161 (1992).

[10] A. v. Oertzen, A. S. Mikhailov, H. H. Rotermund, and G. Ertl, Surf. Sci. 350, 259 (1996).

[11] A. v. Oertzen, A. S. Mikhailov, H. H. Rotermund, and G. Ertl, J. Phys. Chem. B 102, 4966 (1998).

[12] M. Falcke and H. Engel, Phys. Rev. E 50, 1353 (1994).

[13] M. Falcke and H. Engel, J. Chem. Phys. 101, 6255 (1994).

[14] A. v. Oertzen, H. H. Rotermund, A. S. Mikhailov, and G. Ertl, J. Phys. Chem. B 104, 3155 (2000).

[15] H. Schuster, Handbook of Chaos Control (Wiley-VCH, Weinheim, 1999).

[16] E. Ott, C. Grebogi, and J. A. Yorke, Phys. Rev. Lett. 64, 1196 (1990).

[17] W. L. Ditto, S. N. Rauseo, and M. L. Spano, Phys. Rev. Lett. 65, 3211 (1990).

[18] E. R. Hunt, Phys. Rev. Lett. 67, 1953 (1991).

[19] B. Peng, V. Petrov, and K. Showalter, J. Phys. Chem. 95, 4957 (1991).

[20] T. L. Carroll, I. Triandaf, I. Schwartz, and L. Pecora, Phys. Rev. A 46, 6189 (1992).

[21] V. Petrov, V. Gáspár, J. Masere, and K. Showalter, Nature (London) 361, 240 (1993).

[22] S. Bielawski, D. Derozier, and P. Glorieux, Phys. Rev. A 47, 2492 (1993).

[23] I. Triandaf and I. B. Schwartz, Phys. Rev. E 56, 204 (1997).

[24] K. Pyragas, Phys. Lett. A 170, 421 (1992).
[25] D. Gauthier, D. Sukow, H. Concannon, and J. Socolar, Phys. Rev. E 50, 2343 (1994).

[26] J. E. S. Socolar, D. W. Sukow, and D. J. Gauthier, Phys. Rev. E 50, 3245 (1994)

[27] N. Baba, A. Amann, E. Schöll, and W. Just, Phys. Rev. Lett. 89, 074101 (2002).

[28] O. Beck, A. Amann, E. Schöll, J. Socolar, and W. Just, Phys. Rev. E 66, 016213 (2002).

[29] M. Bleich, D. Hochheiser, J. Moloney, and J. Socolar, Phys. Rev. E 55, 2119 (1997).

[30] G. Franceschini, S. Bose, and E. Schöll, Phys. Rev. E 60, 5426 (1999).

[31] T. Pierre, G. Bonhomme, and A. Atipo, Phys. Rev. Lett. 76, 2290 (1996).

[32] P. Parmananda, R. Madrigal, M. Rivera, L. Nyikos, I. Kiss, and V. Gáspár, Phys. Rev. E 59, 5266 (1999).

[33] M. Bertram and A. S. Mikhailov, Phys. Rev. E 63, 066102 (2001).

[34] M. Kim, M. Bertram, M. Pollmann, A. v. Oertzen, A. S. Mikhailov, H. H. Rotermund, and G. Ertl, Science 292, 1357 (2001).

[35] M. Pollmann, M. Bertram, and H. H. Rotermund, Chem. Phys. Lett. 346, 123 (2001).

[36] D. Battogtokh and A. S. Mikhailov, Physica D 90, 84 (1996).

[37] D. Battogtokh, A. Preusser, and A. S. Mikhailov, Physica D 106, 327 (1997).

[38] T. Gritsch, D. Coulman, R. Behm, and G. Ertl, Phys. Rev. Lett. 63, 1086 (1989).

[39] M. Eiswirth and G. Ertl, Surf. Sci. 177, 90 (1986).

[40] H. H. Rotermund, Surf. Sci. Rep. 29, 265 (1997).

[41] H. H. Rotermund, W. Engel, M. Kordesch, and G. Ertl, Nature (London) 343, 355 (1990).

[42] A. v. Oertzen, H. H. Rotermund, and S. Nettesheim, Surf. Sci. 311, 322 (1994).

[43] M. Bertram, Ph.D. thesis, TU Berlin, 2002.

[44] E. M. Izhikevich, Phys. Rev. E 58, 905 (1998).

[45] Y. Kuramoto, Chemical Oscillations, Waves, and Turbulence (Springer, Berlin, 1984).

[46] D. W. Sukow, M. E. Bleich, D. J. Gauthier, and J. E. S. Socolar, Chaos 7, 560 (1997).

[47] W. Just, D. Reckwerth, E. Reibold, and H. Benner, Phys. Rev. E 59, 2826 (1999). 